Monatsschrift f. Geburtshülfe u. Gynäkologie 1935;98:I-IV

\title{
Contents, Vol. 98, 1935
}

Inhaltsverzeichnis.

Originalarbeiten. seite

Atzcrt, W., siehe Guthmann, H.

Barjaktarovic, Svet. Sp., Die Behandlung eitriger Adnexaltumoren

nach meiner Methode 352

Belitz, 0. F, Behandlung von gynäkologischen Kranken mit vaginalen

Moortampons außerhalb der Kurorte 284

Birke, L., Geburtsverletzungen der Nase

144

v. Bud, Georg, Über den Kaiserschnitt in der Längsachse 210

Buschbeck, Herbert, Fortschritte der gynäkologischen Hormon-

therapie $\quad 344$

Chaschinsky, P., und S. Jerschow, Über Metreuryse als Verfahren

der Unterbrechung einer über 12 Wochen dauernden Schwangerschaft 293 Eufinger, H., und

Harald Molz, Die Beeinflussung des Elektro-

kardiogramms durch Schwangerschaft und Geburt 34

-, und J. B. Gottlieb, Pruning der antithyreoidalen Resistenz ver-

schiedener Blutarten im biologischen Versuch

257

Fraymann, S. A., Die Geburtsleitung ohne Dammschutz

Gottlieb, J. B., siehe Eufinger, H.

Guthmann, H., und W. Atzert, Operation oder Strahlenbehandlung

der Myome? Ein Bericht über die in den Jahren 1920 bis 1930 behan-

delten Fälle der Klinik

-, und W. Neuhaus, Mit welcher Genauigkeit erlaubt die Blut-

körperchensenkungsgeschwindigkeit (BSG.) die Beurteilung entzünd-

licher Genitalerkrankungen ? 157

Heimann, Fritz, Die sechsraomentige Mikrosedimentierung der roten

Blutkörperchen und ihre Anwenduug in Gynäkologie und Geburts-

hilfe 266

-, und Felix Schrenk, Das Problem der weiblichen Gonorrhoe (unter besonderer Berücksichtigung ihrer Behandlung) $\quad 10$

Holzapfel, Karl, Gemini permagni 30

-, Beckentrennung oder Sectio caesarea? 31

Honecker, Ludwig, Zur Frage der Typhuseiterung von Ovarialzysten 202

Jerschow, S., siehe Chaschinsky, P.

Junghans, Erich, Primäre Aktinomykose der weiblichen Genitalien

nach Abtreibung 193

Kausch, Fr., Der Einfluß des Tyroxins auf die Blutgerinnung, ein Bei-

trag zu seiner Verwendung als Thromboseprophylaktikum 1

Klaften, E., und R.Wagner, Das Genitalhämatom des Neugeborenen 
als Geburtstrauma 340

Maljawinski, W., Zur Klinik der Uterusrupturen 167

Molz, Harald, siehe Eufinger, H. Neuhaus, W., siehe Guthmann, H.

Randazzo, M., Sterilität und Uterusmyom 270

Schmidt, W. Th., Was können wir zur Herabsetzung der Mortalität

bei innerer Plazentalösung tun? 214

Schmidt, W. Th., Progravid zur Behandlung des Schwangerschafts-

erbrechens 215

YY Inhaltsverzeichnis.

Seite

Schmidt, W. Th., Die Gefühlskälte der Frau und ihre Bekämpfung. . 279

-, Über einen Fall von Vagina duplex 283

Schrenk, Felix, siehe Heimann, Fritz.

Seitz, L., Wann darf eine Frau, die eine Eklampsie oder eine sehwere

Schwangerschaftsnephropatliie durchgemacht hat, wiedcr empf'angen ? 129

Singer, Paul, Em Fall von Graviditas infundibularis 153

Wagner, R., siehe Klaften, E.

Winkier, Hellmuth, Toxische Myokardschädigung im Anschluß an

Präeklampsie 197

W las sow, W., Zur Frage des primären Tubenkarzinoms 217

Grenzgebíete der Geburtshilfe und Gynäkologie.

Physiologie und Krankheiten der Neugeborenen einschließlích derjenigen

der weiblichen Brust. Jahresbericht IV. Von H. Rossenbeck, Gießen 52 Über den heutigen Stand

der Erbbiologie. Von Dr. Hellmuth Winkier,

Frankfurt a. M 85

Psychíatrie, Psychotherapïe, Neurologie (IV). Von Privatdozent Dr. Heinz

Siedentopf, Leipzig 175

Die Röntgentherapie in der Gynäkologie. (Juli 1933 bis Juli 1934.) Von

Franz Wittenbeck, Erlangen 223

Bericht über innersekretorische Literatur (November 1933 bis Oktober 1934).

Von Dr. Hans Dorr, Frankfurt a. M 297

Serologie des Karzinoms und der Schwangerschaft. IV. Jahresbericht. Von

Dr. Erich Tschorne, Graz 362

Siízungsberichíe aus geburtshilflich-gynäkologischen Gesellschaften.

Bericht über die 93. Versammlung der Deutschen Naturforscher und Ärzte

in Hannover. Von Dr. Elisabeth Bechtel, Frankfurt a. M. . . 374 93. Versammlung Deutscher

Naturforscher und Ärzte in Hannover. Vortrag

am 22. September 1934: Über das Hormon des Corpus luteuni von

Ulrich Westphal, Danzig-Langfuhr 315

Gesellschaft für Geburtshílfe und Gynäkologie zu Berlin. Sitzu円g vom 1. Juni

1934102

-, Sitzung vom 20. Juli 1934237

Breslauer Gesellschaft. Sitzung vom 16. Oktober $1934 \quad 312$

Münchener Gynäkologische Gesellschaft. Sitzungen vom 25. Januar uncl

7. Juni 1934180

Nordwestdeutsche Gesellschaft für Geburtshilfe und Gynäkologie. Frühjahrs- 
sitzung und 25. Stiftungsfest am 28. April 1934 in Hamburg .... 241 Mittel- und oberrheinische Gesellschaft für Geburtshílfe und Gynäkologie.

Sitzung am 18. Februar 1934 in Heidelberg 113

Nordostdeutsche Gynäkologische Gesellschaft. Sitzung am 30. Juni und 1. Juli 1934185

Geburtshilflich-Gynäkologische Gesellschaft in Wien. Sitzung vom 12. Juni 1934190

-, Sitzung vom 13. November 1934377

Buchbesprechungen 126, 192, 319, 379

Personalien und Tagesnachrichten 128, 256, 380

W. Tauffer $\dagger \quad 320$ 